\title{
Effect of Sulfur Substitution on Charge Transport Ability of Benzopyrazine-Fused Tetracene Derivatives: A Theoretical Study
}

\author{
Received September 24, 2020; accepted October 12, 2020
}

\author{
Hyunbok Lee* \\ Department of Physics and Institute for Accelerator Science, Kangwon National University, Chuncheon 24341, Republic of Korea
}

*Corresponding author E-mail: hyunbok@kangwon.ac.kr

\begin{abstract}
Atomic substitution in an organic semiconductor alters the intermolecular and intramolecular interactions in the solid state. Thus, an understanding of such electronic interactions is crucial in designing an efficient molecule. In this study, the effect of the substitution of two hydrogen atoms in the tetracene core to sulfur in benzopyrazine-fused tetracene was investigated via theoretical methods. The reorganization energy and electronic coupling of 5,6-substituted benzopyrazine-fused tetracene and its 5,6,11,12-substituted disulfide were calculated using density functional theory. By sulfur substitution, the hole reorganization energy increases but the electron reorganization energy decreases. Meanwhile, the highest values of both hole and electron electronic couplings decrease. As a result, the calculated hole mobility decreased significantly. However, the calculated electron mobility increased slightly, indicating that the charge transport type changes from p-type to ambipolar. These results indicate that atomic substitution significantly affects the charge transport ability of organic semiconductors.
\end{abstract}

Keywords: Density functional theory, Reorganization energy, Electronic coupling, Organic semiconductor

\section{Introduction}

Organic semiconductors are promising materials for a wide range of device applications, including organic light-emitting diodes, organic photovoltaics, and organic field-effect transistors (OFETs) [1]. One of the major advantages of organic semiconductors is their mechanical flexibility [2]. The carrier mobility $(\mu)$ of an organic solid is not significantly hindered upon mechanical bending owing to the weak intermolecular interaction of the van der Waals type. This implies that the $\mu$ of an organic semiconductor can be altered based on the strength of the intermolecular interaction. Hence, an efficient method must be developed to tailor intermolecular interactions for enhancing the device performances of organic semiconductors. In this context, it is evident that atomic substitution changes intermolecular interactions in organic crystals [3-7]. In addition, atomic substitution modifies the charge distribution of a molecule, thereby affecting the intramolecular charge transport properties. Hence, it is important to understand the changes in inter- and intramolecular electronic interactions upon atomic substitution in the design of organic molecules with high $\mu$.

In general, organic semiconductors comprise an expanded structure of aromatic rings such as polyacenes [8]. One of the representative polyacenes is tetracene, which has four aromatic rings. The electronic properties of tetracene can be adjusted by attaching additional functional groups or via the atomic substitution of hydrogen atoms [9]. Such modified materials are beneficial for electronic devices. For example, rubrene, which is a tetracene-derivative with four phenyl groups, exhibits excellent hole mobility $\left(\mu_{\mathrm{h}}\right)$ ranging from 1 to $10 \mathrm{~cm}^{2}$ $\mathrm{V}^{1} \mathrm{~s}^{-1}[10]$. These values are comparable to those of amorphous silicon. Moreover, the substitution of two hydrogen atoms in tetracene to chlorine results in a high $\mu_{\mathrm{h}}$ exceeding $1 \mathrm{~cm}^{2} \mathrm{~V}^{-1} \mathrm{~s}^{-1}$ [11]. These high performances of the devices are attributable to the improvement in the inter- and intramolecular interactions.

Recently, benzopyrazine-fused tetracene derivatives have been suggested as efficient materials for OFETs [12]. The chemical structures of 5,6-substituted benzopyrazine-fused tetracene (TBPy) and its 5,6, 11,12-substituted disulfide (TBPyS) are shown in Fig. 1. By the substitution of two hydrogen atoms in the tetracene core to sulfur, the crystal structure changed significantly. Consequently, the $\mu_{\mathrm{h}}$ of TBPyS was significantly lower than that of TBPy. However, the origin of the

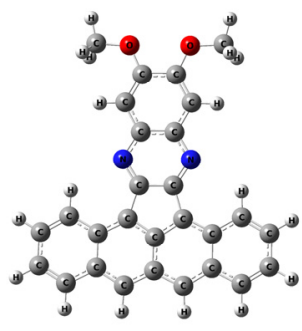

TBPy

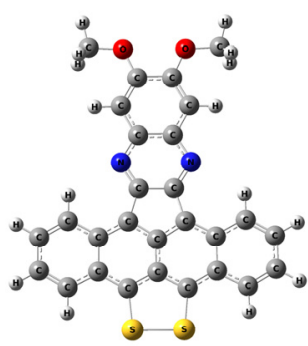

TBPyS
Figure 1. Chemical structures of 5,6-substituted benzopyrazine-fused tetracene (TBPy) and its 5,6,11,12-substituted disulfide (TBPyS). 
different hole transport abilities in TBPy and TBPyS has not been investigated yet.

In this study, the charge transport properties of TBPy and TBPyS were investigated via theoretical calculations. The reorganization energy $(\lambda)$ and electronic coupling $(V)$ were evaluated via the density functional theory (DFT) method. Based on the Marcus theory [13], the $\mu_{h}$ and electron mobility $\left(\mu_{\mathrm{e}}\right)$ values of TBPy and TBPyS were estimated and compared with each other. The origin of the difference in mobility between TBPy and TBPyS is discussed based on calculated charge transport parameters.

\section{Experimental details}

The theoretical estimation of $\mu$ in organic solids based on the Marcus theory has been widely investigated in recent decades. The detailed theory and method are described in our previous reports and in the literature [14-18]. Briefly, the $\mu$ of organic solids can be estimated using the Einstein relation as follows:

$$
\mu=\frac{e}{k_{B} T} D
$$

where $e$ denotes the elementary charge; $k_{\mathrm{B}}$, the Boltzmann constant; $T$, the temperature in Kelvin; and $D$, the diffusion coefficient.

The average $D$ can be estimated as follows:

$$
\mathrm{D}=\frac{1}{2 N} \sum_{i} r_{i}^{2} k_{i} P_{i}
$$

where $N$ denotes the dimensionality; $r_{\mathrm{i}}$, the distance between the molecules in the $\mathrm{i}$-th hopping pathway (PW); $k_{\mathrm{i}}$, the charge hopping rate for the i-th PW; and $P_{\mathrm{i}}$, the hopping probability for the i-th PW.

$k_{\mathrm{i}}$ and $P_{\mathrm{i}}$ can be expressed as follows:

$$
\begin{gathered}
k_{i}=\frac{4 \pi^{2}}{h} V^{2} \frac{1}{\sqrt{4 \pi \lambda k_{B} T}} \exp \left(-\frac{\lambda}{4 k_{B} T}\right) \\
P_{i}=\frac{k_{i}}{\sum_{j} k_{j}},
\end{gathered}
$$

where $h$ denotes the Plank constant. Hence, $V$ and $\lambda$ are the main parameters that determine the charge transport ability of an organic semiconductor. To increase $\mu$, a larger $V$ but a smaller $\lambda$ is required.

$V$ can be calculated as follows:

$$
\begin{aligned}
& \mathrm{V}=\left|\frac{t_{12}-\frac{1}{2}\left(\varepsilon_{1}+\varepsilon_{2}\right) S_{12}}{1-S_{12}}\right| \\
& t_{12}=\left\langle\psi_{1}^{\text {HOMO } / \text { LUMO }}|\hat{F}| \psi_{2}^{\text {HOMO/LUMO }}\right\rangle \\
& S_{12}=\left\langle\psi_{1}^{H O M O / L U M O} \mid \psi_{2}^{H O M O / L U M O}\right\rangle \\
& \varepsilon_{1,2}=\left\langle\psi_{1,2}^{H O M O / L U M O}|\hat{F}|_{\psi_{1,2}^{H O M O} / L U M O}\right\rangle,
\end{aligned}
$$

where $t_{12}$ denotes the transfer integral between two molecules; $\hat{F}$, the Fock operator; $S_{12}$, the spatial overlap between two molecules; $\varepsilon_{1,2}$, the site energy of each molecule; and $\Psi_{1,2}^{\mathrm{HOMO} / L U M O}$, the wave function of the initial and final states in which charge transport occurs [highest occupied molecular orbital (HOMO) in hole transport; lowest unoccupied molecular orbital (LUMO) in electron transport].

$\lambda$ can be evaluated using four single-point energies as follows:

$$
\lambda=\left[E_{ \pm}\left(Q_{0}\right)-E_{ \pm}\left(Q_{ \pm}\right)\right]+\left[E_{0}\left(Q_{ \pm}\right)-E_{0}\left(Q_{0}\right)\right],
$$

where $E_{ \pm}\left(Q_{0}\right)$ denotes the charged state energy calculated at the neutral state geometry; $E_{ \pm}\left(Q_{ \pm}\right)$, the charged state energy calculated at the charged state geometry; $E_{0}\left(Q_{ \pm}\right)$, the neutral state energy calculated at the charged state geometry; and $E_{0}\left(Q_{0}\right)$, the neutral state energy calculated at the neutral state geometry. This method requires a relatively low calculation cost but provides qualitatively useful insights for designing an efficient organic semiconductor.

DFT calculations of single-point energy and geometry optimization were performed with a Becke-style three-parameter exchange and the Lee-Yang-Parr correlation (B3LYP) hybrid functional [1921], whereas that of $V$ was performed using the Perdew-Wang (PW91) functional [22] although the same basis set of 6-31G(d) was used. The energetic minima at the relaxed geometry were verified via vibrational frequency analysis. All quantum mechanical calculations were performed using the Gaussian 09 package [23].

\section{Results and discussion}

Figure 2 shows (a) the calculated Kohn-Sham energy levels of the LUMO and HOMO and (b) the electrostatic potential surfaces of TBPy and TBPyS. In Fig. 2(a), the molecular orbitals of TBPy and TBPyS are shown. The LUMO and HOMO shapes of TBPy and TBPyS were similar, but some contributions from the substituted sulfur atoms were observed. In TBPy, the LUMO and HOMO levels were evaluated to be -2.55 and $-5.02 \mathrm{eV}$, respectively. However, in TBPyS, the LUMO and HOMO levels were evaluated to be -2.63 and

(a)

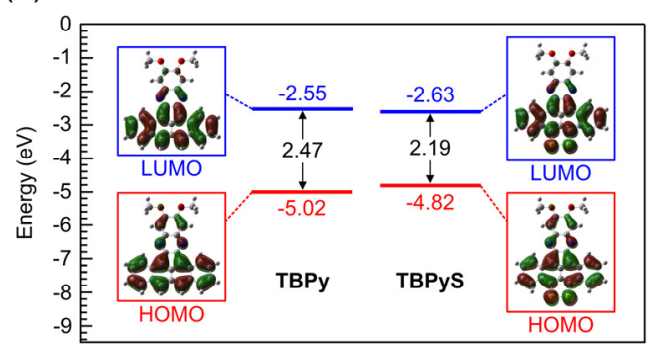

(b)
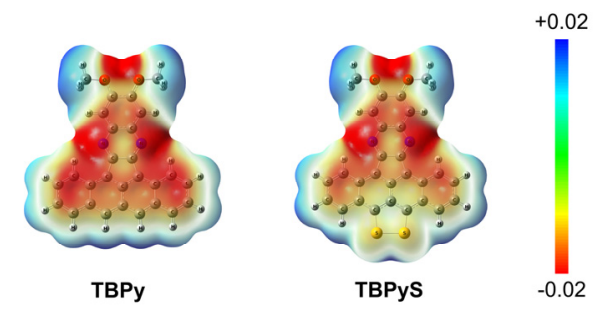

Figure 2. (a) Calculated Kohn-Sham energy levels of lowest unoccupied molecular orbital (LUMO) and highest occupied molecular orbital (HOMO) of TBPy and TBPyS. Corresponding molecular orbitals are shown. (b) Electrostatic potential surface of TBPy and TBPyS (scale bar: $e$ in atomic units). 
$-4.82 \mathrm{eV}$, respectively. Hence, the fundamental gap decreased from 2.47 to $2.19 \mathrm{eV}$ by the substitution of the two hydrogen atoms to sulfur. This can be explained by the broader distribution of wave functions on the molecule. To evaluate the contributions of the sulfur atoms to the LUMO and HOMO, molecular orbital coefficients were calculated. In TBPy, no contribution (0\%) of the two hydrogen atoms in the tetracene core was discovered. However, in TBPyS, the two sulfur atoms contributed to the LUMO and HOMO by $16.4 \%$ and $23.3 \%$, respectively. Hence, the sulfur substitution significantly affected the frontier orbitals that contributed primarily to charge transport. From the perspective of hole injection in OFETs, the energy barrier between the Fermi level of metal electrodes and the HOMO level of an organic semiconductor significant affects the device performance [24]. Considering other p-type organic semiconductors, their HOMO levels calculated at the same level [B3LYP/6-31G(d)] were $-4.86 \mathrm{eV}$ for tetracene, $-4.67 \mathrm{eV}$ for rubrene, and $-4.60 \mathrm{eV}$ for pentacene. Hence, the HOMO level of TBPyS was comparably shallow, but that of TBPy was deeper. This can imply that the formation of an Ohmic contact of TBPy with electrodes may be more difficult than that of TBPyS. Hence, an interlayer with a high work function (e.g., molybdenum trioxide [25]) would be necessary.

As shown in Fig. 2(b), the two hydrogen atoms in TBPy were more positively charged than the two sulfur atoms in TBPyS. To quantitatively evaluate the charge distribution, Mulliken charge analysis was performed for the neutral and charged states. The results indicate that the charge on the hydrogen atom in the neutral, cation, and anion states of TBPy were $0.134684 e, 0.181382 e$, and $0.08363 e$, respectively. Hence, the change in charge between the cation and neutral states was $0.046698 e$, whereas that between the anion and neutral states was $-0.051320 e$. Meanwhile, the charges on the sulfur atom in the neutral, cation, and anion states of TBPyS were $0.124066 e$, $0.235474 e$, and $0.027316 e$, respectively. Therefore, the change in charge between the cation and neutral states was $0.111408 e$, whereas that between the anion and neutral states was -0.096750 e. Accordingly, the changes in charge between the neutral and charged states of TBPyS were larger than those of TBPy. This is attributable to the higher contributions of the sulfur atoms to the LUMO and HOMO.

Table I shows the calculated hole reorganization energy $\left(\lambda_{\mathrm{h}}\right)$ and electron reorganization energy $\left(\lambda_{\mathrm{e}}\right)$ of TBPy and TBPyS. The $\lambda_{\mathrm{h}}$ and $\lambda_{\mathrm{e}}$ of TBPy were 115 and $240 \mathrm{meV}$, respectively, whereas those of TBPyS were 133 and $212 \mathrm{meV}$, respectively. Hence, the sulfur substitution increased $\lambda_{\mathrm{h}}$ but decreased $\lambda_{\mathrm{e}}$. This indicates that the intramolecular hole transport ability was hindered, whereas the intramolecular electron transport ability was enhanced. This trend is similar to that observed in chlorine-substituted pentacene derivatives [26], in which fluorine substitution increased $\lambda_{\mathrm{h}}$ and $\lambda_{\mathrm{e}}$, whereas chlorine substitution increased $\lambda_{\mathrm{h}}$ but decreased $\lambda_{\mathrm{e}}$. This was reported to be due to the formation of $\pi$-orbitals between the vacant $d$-orbital of chlorine and the pentacene backbone. This interaction would cause less molecular distortions during electron gain, resulting in less energy change. Comparing the current case, sulfur has a vacant d-orbital; hence, the same explanation is applicable for sulfur substitution. However, the detailed mechanism should be investigated further. Meanwhile, to understand the effect of the polarization functions of hydrogen atoms on $\lambda_{\mathrm{h}}$ and $\lambda_{\mathrm{e}}$, the same calculations were performed with basis set 6$31 \mathrm{G}(\mathrm{d}, \mathrm{p})$. However, all the values were identical, indicating that the addition of polarization functions to hydrogen atoms did not affect $\lambda_{\mathrm{h}}$ and $\lambda_{\mathrm{e}}$.
Table I. Hole and electron reorganization energies $\left(\lambda_{h}\right.$ and $\lambda_{\mathrm{e}}$, respectively) of TBPy and TBPyS.

\begin{tabular}{lcc}
\hline & TBPy & TBPyS \\
\hline$\lambda_{\mathrm{h}}(\mathrm{meV})$ & 115 & 133 \\
$\lambda_{\mathrm{e}}(\mathrm{meV})$ & 240 & 212 \\
\hline
\end{tabular}

(a) TBPy



(b) TBPyS



Figure 3. Possible hopping pathway (PW) of (a) TBPy and (b) TBPyS in crystal structure. Reference molecule is colored in red; PWs with nearest neighbor molecules are numbered. Hydrogen atoms are omitted for simplicity.

The possible hopping PWs of TBPy and TBPyS in the crystal structure are shown in Fig. 3 and related parameters are presented in Tables II and III. The reference molecule is colored in red, whereas PWs with nearest neighbor molecules are numbered. The crystal structures were obtained from the crystallographic information file in the literature [12]. $d$ was calculated based on the center of mass between the molecules. In TBPy, the PWs with the shortest $d$ were PW1 and PW8, both of which had the same intermolecular position. The $d$ values of PW1 and PW8 were $5.276 \AA$, in which the hole electronic coupling $\left(V_{\mathrm{h}}\right)$ and electron electronic coupling $\left(V_{\mathrm{e}}\right)$ were -84.81 and $96.43 \mathrm{meV}$, respectively. Consequently, the hole hopping probability $\left(P_{\mathrm{h}}\right)$ and electron hopping probability $\left(P_{\mathrm{e}}\right)$ indicated the highest values of $49.5 \%$ and $48.3 \%$, respectively. Hence, the holes and electrons were primarily transported via PW1 and PW8. The contributions from the other PWs to charge transport were marginal.

Meanwhile, in TBPyS, the PWs with the shortest $d$ were PW1 and PW6, both of which had the same intermolecular position. However, the highest values of $V_{\mathrm{h}}$ and $V_{\mathrm{e}}$ were discovered in PW7. In PW1 and PW6, $d$ was $3.892 \AA$, in which $V_{\mathrm{h}}$ and $V_{\mathrm{e}}$ were 24.74 and $-50.31 \mathrm{meV}$, respectively. However, in PW7, $d$ was $12.449 \AA$, in which $V_{\mathrm{h}}$ and $V_{\mathrm{e}}$ were 32.04 and $58.40 \mathrm{meV}$, respectively. These high values, despite the long $d$, were due to the unconventional intermolecular interaction via the sulfur atoms in PW7. It has been reported that sulfur-sulfur interactions can provide an efficient pathway for charge transport in hexathiapentacene [27], and a similar effect was estimated 
Table II. Molecular distance $(d)$, calculated hole and electron electronic couplings $\left(V_{\mathrm{h}}\right.$ and $V_{\mathrm{e}}$, respectively), and hole and electron hopping probabilities $\left(P_{\mathrm{h}}\right.$ and $P_{\mathrm{e}}$, respectively) for each PW of TBPy.

\begin{tabular}{lcccccccccccc}
\hline PW & 1 & 2 & 3 & 4 & 5 & 6 & 7 & 8 & 9 & 10 & 11 & 12 \\
\hline$d(\AA)$ & 5.276 & 11.831 & 8.694 & 14.641 & 8.694 & 12.277 & 12.277 & 5.276 & 9.951 & 11.808 & 9.951 & 11.831 \\
$V_{\mathrm{h}}(\mathrm{meV})$ & -84.81 & -6.55 & -2.48 & -0.45 & -2.48 & -0.05 & -0.05 & -84.81 & -1.86 & -5.32 & -1.86 & -6.55 \\
$V_{\mathrm{e}}(\mathrm{meV})$ & 96.43 & -3.45 & 17.59 & 2.26 & 17.59 & -0.13 & -0.13 & 96.43 & -0.33 & -2.13 & -0.33 & -3.45 \\
$P_{\mathrm{h}}(\%)$ & 49.5 & 0.3 & 0.0 & 0.0 & 0.0 & 0.0 & 0.0 & 49.5 & 0.0 & 0.2 & 0.0 & 0.3 \\
$P_{\mathrm{e}}(\%)$ & 48.3 & 0.1 & 1.6 & 0.0 & 1.6 & 0.0 & 0.0 & 48.3 & 0.0 & 0.0 & 0.0 & 0.1 \\
\hline
\end{tabular}

Table III. Molecular distance (d), calculated hole and electron electronic couplings ( $V_{\mathrm{h}}$ and $V_{\mathrm{e}}$, respectively), and hole and electron hopping probabilities $\left(P_{\mathrm{h}}\right.$ and $P_{\mathrm{e}}$, respectively) for each PW of TBPyS.

\begin{tabular}{|c|c|c|c|c|c|c|c|c|c|c|}
\hline PW & 1 & 2 & 3 & 4 & 5 & 6 & 7 & 8 & 9 & 10 \\
\hline$d(\AA)$ & 3.892 & 11.108 & 11.689 & 13.557 & 13.571 & 3.892 & 12.449 & 11.689 & 11.108 & 13.557 \\
\hline$V_{\mathrm{h}}(\mathrm{meV})$ & 24.74 & -0.87 & -0.06 & -0.19 & 2.19 & 24.74 & 32.04 & -0.06 & -0.87 & -0.19 \\
\hline$V_{\mathrm{e}}(\mathrm{meV})$ & -50.31 & -0.23 & -0.12 & -1.15 & -0.56 & -50.31 & 58.40 & -0.12 & -0.23 & -1.15 \\
\hline$P_{\mathrm{h}}(\%)$ & 27.1 & 0.0 & 0.0 & 0.0 & 0.2 & 27.1 & 45.5 & 0.0 & 0.0 & 0.0 \\
\hline$P_{\mathrm{e}}(\%)$ & 29.9 & 0.0 & 0.0 & 0.0 & 0.0 & 29.9 & 40.2 & 0.0 & 0.0 & 0.0 \\
\hline
\end{tabular}

Table IV. Calculated hole and electron mobilities ( $\mu_{\mathrm{h}}$ and $\mu_{\mathrm{e}}$, respectively) of TBPy and TBPyS.

\begin{tabular}{lcc}
\hline & TBPy & TBPyS \\
\hline$\mu_{\mathrm{h}}\left(\mathrm{cm}^{2} \mathrm{~V}^{-1} \mathrm{~s}^{-1}\right)$ & 2.08 & 0.63 \\
$\mu_{\mathrm{e}}\left(\mathrm{cm}^{2} \mathrm{~V}^{-1} \mathrm{~s}^{-1}\right)$ & 0.54 & 0.71 \\
\hline
\end{tabular}

in the TBPyS case. PW1 and PW6 indicated the next highest $P_{\mathrm{h}}$ and $P_{\mathrm{e}}$. However, except for PW1, PW6, and PW7, charge transport barely occurred.

Using the calculated charge transport parameters, the theoretical $\mu_{\mathrm{h}}$ and $\mu_{\mathrm{e}}$ of TBPy and TBPyS were estimated as shown in Table IV. The $\mu_{\mathrm{h}}$ and $\mu_{\mathrm{e}}$ of TBPy were evaluated as 2.08 and $0.54 \mathrm{~cm}^{2} \mathrm{~V}^{-1} \mathrm{~s}^{-1}$, respectively. However, owing to sulfur substitution, $\mu_{\mathrm{h}}$ decreased significantly to $0.63 \mathrm{~cm}^{2} \mathrm{~V}^{-1} \mathrm{~s}^{-1}$. This decrease in $\mu_{\mathrm{h}}$ was consistent with the experimentally measured $\mu_{\mathrm{h}}$ in the literature [12]. However, the calculated $\mu_{\mathrm{h}}$ was much higher than the measured value of $\mu_{\mathrm{h}}$. This may be because extrinsic factors affected $\mu_{\mathrm{h}}$, e.g., charge trapping by defects and contact formation with electrodes. Nevertheless, the change in $\mu_{\mathrm{h}}$ due to sulfur substitution can be well described qualitatively: the decrease in $\mu_{\mathrm{h}}$ was caused by both the increase in $\lambda_{\mathrm{h}}$ and the decrease in $V_{\mathrm{h}}$. Meanwhile, $\mu_{\mathrm{e}}$ increased slightly to $0.71 \mathrm{~cm}^{2} \mathrm{~V}^{-1} \mathrm{~s}^{-1}$. Consequently, the ratio of $\mu_{\mathrm{h}}$ to $\mu_{\mathrm{e}}$ was close to unity, indicating that the charge transport type changed from p-type to ambipolar by sulfur substitution. Such ambipolar characteristics may be useful for applications involving inverter logic gates and organic light-emitting transistors [28].

\section{Conclusions}

In this study, the effect of the substitution of hydrogen atoms in the tetracene core to sulfur was investigated via theoretical calculations. By sulfur substitution, $\lambda_{\mathrm{h}}$ increased from 115 to $133 \mathrm{meV}$, whereas $\lambda_{\mathrm{e}}$ decreased from 240 to $212 \mathrm{meV}$. In TBPy, the highest values of $V_{\mathrm{h}}$ and $V_{\mathrm{e}}$ were -84.81 and $96.43 \mathrm{meV}$, respectively. However, in TBPyS, the highest values of $V_{\mathrm{h}}$ and $V_{\mathrm{e}}$ were 32.04 and $58.40 \mathrm{meV}$, respectively. Consequently, the $\mu_{\mathrm{h}}$ and $\mu_{\mathrm{e}}$ of TBPy were calculated to be 2.08 and $0.54 \mathrm{~cm}^{2} \mathrm{~V}^{-1} \mathrm{~s}^{-1}$, respectively, whereas those of TBPyS were 0.63 and $0.71 \mathrm{~cm}^{2} \mathrm{~V}^{-1} \mathrm{~s}^{-1}$, respectively. Hence, the sulfur substitution deteriorated hole transport in electronic devices owing to both the increase in $\lambda_{\mathrm{h}}$ and the decrease in $V_{\mathrm{h}}$. Meanwhile, the charge transport type of TBPy changed from p-type to ambipolar by sulfur substitution owing to the modified intra- and intermolecular electronic interactions. These results demonstrate the substantial effect of atomic substitution in organic semiconductors on device performance.

\section{Acknowledgements}

This study was supported by the National Research Foundation of Korea (NRF-2018R1D1A1B07051050 and 2018R1A6A1A03025582).

\section{References}

[1] F. So, Organic Electronics: Materials, Processing, Devices and Applications (CRC Press, Boca Raton, 2010).

[2] S. E. Root, S. Savagatrup, A. D. Printz, D. Rodriquez, and D. J. Lipomi, Chem. Rev. 117, 6467 (2017).

[3] G. Zhao, H. Dong, H. Zhao, L. Jiang, X. Zhang, J. Tan, Q. Meng, and W. Hu, J. Mater. Chem. 22, 4409 (2012).

[4] H. T. Black, S. Liu, and V. S. Ashby, Org. Lett. 13, 6492 (2011).

[5] J. Zhang, Z. Ma, Q. Zhang, T. S. Virk, H. Geng, D. Wang, W. Xu, Z. Shuai, K. Singh, W. Hu, and D. Zhu, J. Mater. Chem. C 1, 5765 (2013).

[6] O. Pitayatanakul, K. Iijima, M. Ashizawa, T. Kawamoto, H. Matsumoto, and T. Mori, J. Mater. Chem. C 3, 8612 (2015).

[7] T. Kadoya, S. Mano, A. Hori, K. Tahara, K. Sugimoto, K. Kubo, M. Abe, H. Tajima, and J. I. Yamada, Org. Electron. 78, 105570 (2020).

[8] J. E. Anthony, Angew. Chem. Int. Ed. 47, 452 (2008).

[9] C. Goldmann, S. Haas, C. Krellner, K. P. Pernstich, D. J. Gundlach, and B. Batlogg, J. Appl. Phys. 96, 2080 (2004). 
[10] M. A. Reyes-Martinez, A. J. Crosby, and A. L. Briseno, Nat. Commun. 6, 6948 (2015).

[11] H. Moon, R. Zeis, E. J. Borkent, C. Besnard, A. J. Lovinger, T. Siegrist, C. Kloc, and Z. Bao, J. Am. Chem. Soc. 126, 15322 (2004).

[12] S. Kojima, T. Okamoto, K. Miwa, H. Sato, J. Takeya, and Y. Matsuo, Org. Electron. 14, 437 (2013).

[13] R. A. Marcus, Rev. Mod. Phys. 65, 599 (1993).

[14] H. Lee, K. Jeong, S. W. Cho, and Y. Yi, J. Chem. Phys. 137, 034704 (2012).

[15] H. Lee, Y. Yi, S. W. Cho, and W. K. Choi, Synth. Met. 194, 118 (2014).

[16] H. Lee, Sci. Adv. Mater. 10, 989 (2018).

[17] Z. Shuai, L. Wang, and C. Song, Theory of Charge Transport in Carbon Electronic Materials, (Springer, Heidelberg, 2012).

[18] H. Lee, and H. Lee, New Phys. -Sae Mulli 67, 1193 (2017).

[19] A. D. Becke, J. Chem. Phys. 98, 5648 (1993).

[20] C. Lee, W. Yang, and R. G. Parr, Phys. Rev. B 37, 785 (1988).

[21] S. F. Sousa, P. A. Fernandes, and M. J. Ramos, J. Phys. Chem. A 111, 10439 (2007).

[22] J. P. Perdew, J. A. Chevary, S. H. Vosko, K. A. Jackson, M. R. Pederson, D. J. Singh, and C. Fiolhais, Phys. Rev. B 46, 6671 (1992).

[23] M. J. Frisch, G. W. Trucks, H. B. Schlegel, G. E. Scuseria, M. A. Robb, J. R. Cheeseman, G. Scalmani, V. Barone, B. Mennucci, G.
A. Petersson, H. Nakatsuji, M. Caricato, X. Li, H. P. Hratchian, A. F. Izmaylov, J. Bloino, G. Zheng, J. L. Sonnenberg, M. Hada, M. Ehara, K. Toyota, R. Fukuda, J. Hasegawa, M. Ishida, T. Nakajima, Y. Honda, O. Kitao, H. Nakai, T. Vreven, J. A. J. Montgomery, J. E. Peralta, F. Ogliaro, M. Bearpark, J. J. Heyd, E. Brothers, K. N. Kudin, V. N. Staroverov, R. Kobayashi, J. Normand, K. Raghavachari, A. Rendell, J. C. Burant, S. S. Iyengar, J. Tomasi, M. Cossi, N. Rega, J. M. Millam, M. Klene, J. E. Knox, J. B. Cross, V. Bakken, C. Adamo, J. Jaramillo, R. Gomperts, R. E. Stratmann, O. Yazyev, A. J. Austin, R. Cammi, C. Pomelli, J. W. Ochterski, R. L. Martin, K. Morokuma, V. G. Zakrzewski, G. A. Voth, P. Salvador, J. J. Dannenberg, S. Dapprich, A. D. Daniels, Ö. Farkas, J. B. Foresman, J. V. Ortiz, J. Cioslowski, and D. J. Fox, Gaussian 09, Gaussian Inc., Wallingford CT, 2009.

[24] H. Lee, S. W. Cho, and Y. Yi, Curr. Appl. Phys. 16, 1533 (2016).

[25] Y. Yi, P. E. Jeon, H. Lee, K. Han, H. S. Kim, K. Jeong, and S. W. Cho, J. Chem. Phys. 130, 094704 (2009).

[26] J. X. Fan, L. F. Ji, N. X. Zhang, P. P. Lin, G. Y. Qin, S. F. Zhang, and A. M. Ren, New J. Chem. 43, 3583 (2019).

[27] A. L. Briseno, Q. Miao, M. M. Ling, C. Reese, H. Meng, Z. Bao, and F. Wudl, J. Am. Chem. Soc. 128, 15576 (2006).

[28] Y. Ren, X. Yang, L. Zhou, J. Y. Mao, S. T. Tan, and Y. Zhou, Adv. Funct. Mater. 29, 1902105 (2019). 